\section{Nickelallergie - unsichere Diagnose bei Babys und Kleinkindern}

Viele Kleinkinder tragen bereits Schmuck. Entsprechend besteht die Gefahr einer Nickelallergie. Epikutantests liefern im frühen Kindesalter jedoch noch keine zuverlässigen Daten wie dänische Wissenschaftler jetzt zeigen konnten.

n einer Voruntersuchung wurden 562 Säuglinge und Kleinkinder einer Epikutantestung mit Nickelsulfat unterzogen. Der Test wurde im Alter von drei, sechs, zwölf und 18 Monaten wiederholt. Fiel dieser bei den zwölf und 18 Monate alten Kleinkindern positiv aus, galt eine Nickelsensibilisierung als bewiesen. Dies traf auf 26 Kinder $(8,6 \%)$ zu. Bei 111 Kindern fielen die Tests über den Beobachtungszeitraum hinweg wechselhaft positiv und negativ aus. Unter allen positiv Getesteten war lediglich ein Fall von klinischer Relevanz.

Aus der Gruppe der 26 Nickel-sensibilisierten Kinder wurde bei 24 Kindern im Alter von drei Jahren ein Wiederholungstest durchgeführt. Sieben Kinder reagierten erneut positiv, 16 negativ (ein Ausschluss). Die verbliebenen 23 Kinder wurden im Alter von sechs Jahren noch einmal getestet: Fünf Kinder reagierten positiv, 18 negativ. Unter den anfangs 26 auffälligen Kindern fand sich bei den Sechsjährigen lediglich noch eine klinisch relevante Nickelsensibilisierung. Nur zwei von 21 Kindern, die sowohl mit zwölf und 18 Monaten als auch mit drei ten reproduzierbare Testreaktionen.

Fazit: Die Untersuchungen zeigen, dass Nickelsensibilisierungen bereits in früher Kindheit vorkommen. Eine Epikutantestung ist schon in diesem Lebensalter möglich. Eingeschränkt wird deren diagnostischer Wert jedoch durch eine höhere Zahl falsch positiver Hautreaktionen. Falsch negative dürften ebenfalls vorkommen, wurden aber in der Arbeit nicht quantifiziert. Für die Praxis ist zu empfehlen, sich bei Verdacht auf Konund sechs Jahren getestet wurden, hat-

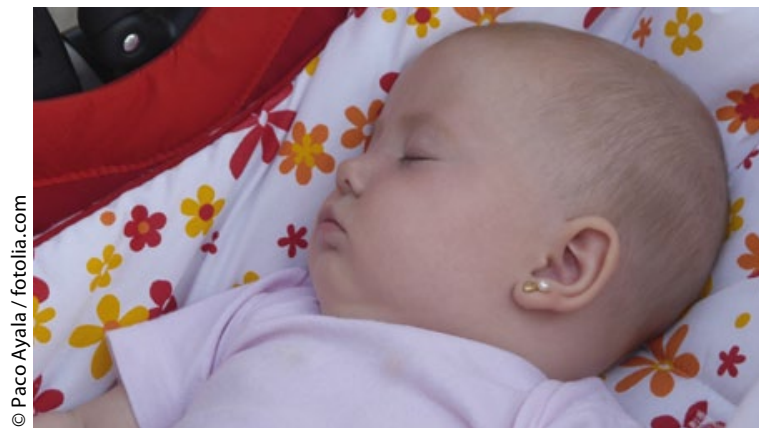

Schmuck im frühen Kindesalter wie z. B. Ohrringe gilt als eine der Hauptquellen für Nickelsensibilisierungen.

taktsensibilisierung gegenüber Nickel im Kleinkindalter zunächst auf Anamnese und Befund zu stützen und eine Epikutantestung auf Nickel nur dann durchzuführen, wenn diese unabdingbar scheint. Die Arbeit zeigt einmal mehr, dass aus Gründen der Prophylaxe Hautkontakt mit nickelhaltigem Metall vermieden werden sollte.

Dr. Dieter Bruchhausen

Mortz CG et al. Positive nickel patch tests in infants are of low clinical relevance and rarely reproducible. Pediatr Allergy Immunol 2013; 24: $84-7$

\title{
Anaphylaxie: Adrenalin inhalieren statt injizieren?
}

Bei lebensbedrohlichen allergischen Reaktionen ist Adrenalin i.m. das wichtigste Medikament. Viele Patienten scheuen jedoch den Einsatz des Autoinjektors. Ist hier Inhalation eine probate Alternative?

$P$ hari harmakologen der Universität Köln haben untersucht, ob die Inhalation von vernebeltem Adrenalin für die Selbstmedikation geeignet ist. Bei acht gesunden Probanden wurden Pharmakokinetik und Pharmakodynamik von vernebeltem inhaliertem Adrenalin in zwei Dosierungen (4 mg und $8 \mathrm{mg}$ ) mit denen von i.m. injiziertem Adrenalin $(0,3 \mathrm{mg})$ und denen einer inhalierten Placebolösung verglichen.

Nach der Anwendung stiegt der Adrenalin-Plasmaspiegel in allen drei Gruppen rasch an: Die maximale Konzentration $\left(C_{\max }\right)$ wurde innerhalb von etwa 20 Minuten erreicht. Nach 45-60 Minuten war der Spiegel bei den meisten Probanden auf das Ausgangsniveau zurückgefallen. Die mittlere Adrenalin-Exposition war mit der 8-mg-Dosis inhalativen Adrenalins am höchsten, gefolgt von der 4-mg-Dosis und dem I.-m.-Adrenalin, wobei alle Werte signifikant höher lagen als bei der Placebotherapie.

Die Bioäquivalenz von inhaliertem und injiziertem Adrenalin konnte trotzdem nicht bewiesen werden. Wegen der großen Schwankungsbreite von $\mathrm{C}_{\max }{ }^{-}$ und AUC-Werten (Konzentrations-ZeitKurve) waren relevante Unterschiede nicht auszuschließen. „Trotzdem weisen die AUC-Werte darauf hin, dass die Inhalation von $8 \mathrm{mg}$ Adrenalin der I.-m.Injektion von $0,3 \mathrm{mg}$ Adrenalin nicht unterlegen ist“, schreiben die Studienautoren unter Leitung von Prof. Dr. Uwe Fuhr.

Als Reaktion auf die Verumtherapien erhöhte sich die Herzfrequenz, und zwar signifikant stärker mit den inhalativen Therapien als mit der I.-m.-Gabe. Die Unterschiede wurden gemäß vordefinierter Grenzwerte jedoch als nicht relevant eingestuft. Die anfängliche Herzfrequenz war nach etwa einer Stunde wieder erreicht. Die Studienmedikation wurde insgesamt gut vertragen.

Fazit: Die Pilotstudie zeigt, dass die Inhalation von vernebeltem Adrenalin über ein tragbares Gerät die Substanz im Blutkreislauf verfügbar macht. „Mit 4 oder $8 \mathrm{mg}$ inhalativem Adrenalin werden im Vergleich zu 0,3 mg Adrenalin i. m. eine mindestens ebenso hohe systemische Exposition und zudem stärkere hämodynamische Effekte erzielt, und das bei einem ähnlichen Sicherheitsprofil“, schreiben die Kölner Pharmakologen. Dr. Beate Schumacher

Breuer $\mathrm{C}$ et al. Pharmacokinetics and pharmacodynamics of moist inhalation epinephrine using a mobile inhaler. Eur J Clin Pharmacol 2013; 69: 1303-10 